\title{
Atezolizumab and Bevacizumab in Patients with Unresectable Hepatocellular Carcinoma: Pharmacokinetic and Safety Assessments Based on Hepatic Impairment Status and Geographic Region
}

\author{
Colby S. Shemesh ${ }^{\mathrm{a}}$ Phyllis Chan ${ }^{\mathrm{a}}$ Hui Shao ${ }^{\mathrm{b}}$ Derek-Zhen $\mathrm{Xu}^{\mathrm{c}}$ Daniel Combs ${ }^{\mathrm{a}}$ \\ Shweta Vadhavkara René Bruno ${ }^{d}$ Benjamin Wua \\ ${ }^{a}$ Department of Clinical Pharmacology, Genentech Inc., South San Francisco, CA, USA; b Safety Science, F. Hoffmann- \\ La Roche Ltd., Beijing, China; 'Product Development Oncology, F. Hoffmann-La Roche Ltd., Shanghai, China; ${ }^{d}$ Clinical \\ Pharmacology, Genentech-Roche, Marseille, France
}

\section{Keywords}

Atezolizumab - Bevacizumab - Hepatic impairment .

Geographic region · Clinical pharmacology

\begin{abstract}
Introduction: Phase 1b G030140 and phase 3 IMbrave150 studies evaluated first-line atezolizumab + bevacizumab for unresectable hepatocellular carcinoma (HCC). Here, we evaluated pharmacokinetics (PK) and safety by hepatic impairment status and geographic region. Methods: Patients received atezolizumab 1,200 mg + bevacizumab $15 \mathrm{mg} / \mathrm{kg}$ IV every 3 weeks. Drug concentrations were evaluated by descriptive statistics and population PK. PK and adverse event frequencies were evaluated by hepatic impairment status and region. Results: 323 IMbrave150 patients and 162 GO30140 patients were PK evaluable. Compared with IMbrave150 patients who had normal hepatic function per the National Cancer Institute Organ Dysfunction Working Group (NCl-ODWG) criteria $(n=123)$, patients with mild impairment $(n=171)$ had a geometric mean ratio (GMR) of 0.92 for cycle 1 atezolizumab area under the concentration-time curve (AUC); patients with moderate impairment $(n=27)$ had a
\end{abstract}

GMR of 0.88. Patients in Asia ([ $n=162]$ vs. outside [ $n=161]$ ) had a GMR of 1.25 for cycle 1 atezolizumab AUC. Compared with GO30140 patients who had normal hepatic function ( $\mathrm{NCl}-\mathrm{ODWG}[n=61])$, patients with mild impairment $(n=92)$ had a GMR of 0.97 for cycle 1 peak bevacizumab concentrations; those with moderate impairment $(n=9)$ had a GMR of 0.94. Patients in Asia $(n=111)$ versus outside Asia $(n=51)$ had a GMR of 0.94 for cycle 1 peak bevacizumab concentration. PK results were generally comparable when evaluated based on additional hepatic functional definitions (ChildPugh or albumin/bilirubin criteria) or study enrollment in Japan. No associations between atezolizumab PK and HCC etiology were seen. Adverse event frequencies were similar across evaluated groups. Conclusions: IMbrave150 and GO30140 patients with unresectable HCC had varying baseline hepatic impairment and high enrollment from Asia. PK data demonstrated considerable exposure overlap across groups. Treatment was tolerable across groups. No need for dose adjustment based on mild or moderate hepatic impairment or region is recommended based on this analysis.

(c) 2021 The Author(s)

Published by S. Karger AG, Basel karger@karger.com www.karger.com/lic

Karger $\stackrel{\text { ' }}{5}$

GOPEN ACCESS
(C) 2021 The Author(s)

Published by S. Karger AG, Basel

This is an Open Access article licensed under the Creative Commons Attribution-NonCommercial-4.0 International License (CC BY-NC) (http://www.karger.com/Services/OpenAccessLicense), applicable to the online version of the article only. Usage and distribution for commercial purposes requires written permission.
Correspondence to:

Colby S. Shemesh, shemesh.colby@gene.com 


\section{Introduction}

Hepatocellular carcinoma (HCC) is a medically complex and lethal disease with rising incidence, poor prognosis, and, thus, high unmet medical need [1]. HCC is the fourth leading cause of cancer-related mortality worldwide $[2,3]$. Patients with HCC present with inconspicuous symptoms and are generally diagnosed at a late stage, often eliminating possibilities for local curative treatments [4]. The global standard of care for unresectable or advanced HCC has included the use of tyrosine kinase inhibitors such as sorafenib; however, only limited responses are achieved [5], and sorafenib, for one, prolongs the overall survival by only 3 months compared with placebo [4].

Atezolizumab, an agent targeted against programmed death-ligand 1, and bevacizumab, which targets vascular endothelial growth factor, together make a unique cancer immunotherapy doublet option for patients with HCC. The pairing allows for normalization of tumor vasculature, retraction of an immunosuppressive tumor microenvironment, and reprogramming of immune checkpoints to enhance stimulation and infiltration of immune cells with T-cell activation in the tumor [6-9]. IMbrave150 (NCT03434739) is a phase 3 global, randomized, controlled trial comparing first-line atezolizumab plus bevacizumab with the multikinase inhibitor sorafenib in patients with HCC [10]. In IMbrave150, a statistical and clinically meaningful overall survival and progression-free suvival improvement was seen over sorafenib when atezolizumab was administered in combination with bevacizumab in the first-line setting for unresectable HCC. Consistent with data from the phase I GO30140 study [11], the combination was also generally well tolerated, with manageable toxicities, and the safety profile was consistent with the known risks of the individual study treatments. Atezolizumab plus bevacizumab received market approval in 2020 [10].

Despite extensive characterization of the clinical pharmacology properties of atezolizumab [12-15] and bevacizumab [16], pharmacokinetic (PK) evaluation of the combination in HCC has not been reported, nor have data by hepatic function. As hepatic metabolism does not contribute $>20 \%$ to the metabolism of a monoclonal antibody $(\mathrm{mAb})$, dedicated studies of hepatic impairment effects on PK during $\mathrm{mAb}$ clinical development had generally not been considered required [17]. However, limited data published in 2020 revealed a trend for exposure decrease with several mAbs in patients with hepatic impairment [18]. Reports have also indicated that the clearance of certain $\mathrm{mAbs}$ was faster in patients who received liver transplants, likely due to the additional clearance route of asci- tes fluid draining $[19,20]$. At the time of this writing, several approved checkpoint inhibitors, such as ipilimumab, pembrolizumab, and durvalumab, do not have dosing instructions in the package insert for patients with moderate hepatic impairment [21]. As hepatic impairment is a common occurrence in patients with HCC, and may increase as the disease progresses [22], knowledge of the effects of hepatic impairment on the PK and safety of agents used in HCC is essential to ensure correct dosing.

Another important characteristic pertaining to drug exposure includes investigation of regional and ethnic differences such as disease prevalence and target biology, which could give rise to variation in FCGRT gene expression and $\mathrm{FC} \gamma \mathrm{R}$ expression, as well as variations in immune response that might influence the clearance of monoclonal antibodies [23-27]. Given the importance of potential differences across ethnic factors, a framework for evaluating the impact on the benefit-risk profile of new therapeutic agents exists [28-30]. In many cases, exposure is extrapolated from White patients in landmark studies to Asian populations for clinical use [31], with PK bridging studies being routinely requested by regulatory agencies in Asia for new therapies [32]. In HCC, racial and/or ethnic differences in tumor stage and severity of liver disease at diagnosis have been observed [33-35] and could potentially give rise to clinical characteristics impacting drug exposure, safety, and efficacy. Additionally, such factors are influential on the etiology of HCC, with hepatitis $\mathrm{C}$ virus being the most frequent viral cause in Western countries and Japan, and hepatitis B virus being the predominant viral factor in other Asian countries [36]. Ethnicity (race and region) was evaluated among covariates in a number of population PK (popPK) studies in bevacizumab and atezolizumab and was not found to influence the PK of either $[12,16]$; however, no patients with HCC were included in these analyses.

In this article we report both PK - including exposure metrics - as well as safety findings for atezolizumab + bevacizumab in patients with HCC, evaluated by hepatic impairment status and global region. We also evaluated potential associations of atezolizumab PK with HCC etiology (hepatitis B virus, hepatitis C virus, or nonviral).

\section{Materials and Methods}

\section{Contributing Studies}

IMbrave150 is a global, phase 3, open-label, randomized study evaluating the efficacy and safety of atezolizumab with bevacizumab versus sorafenib in patients with unresectable HCC who have not received prior systemic treatment. Primary data from this 
a) IMbrave150

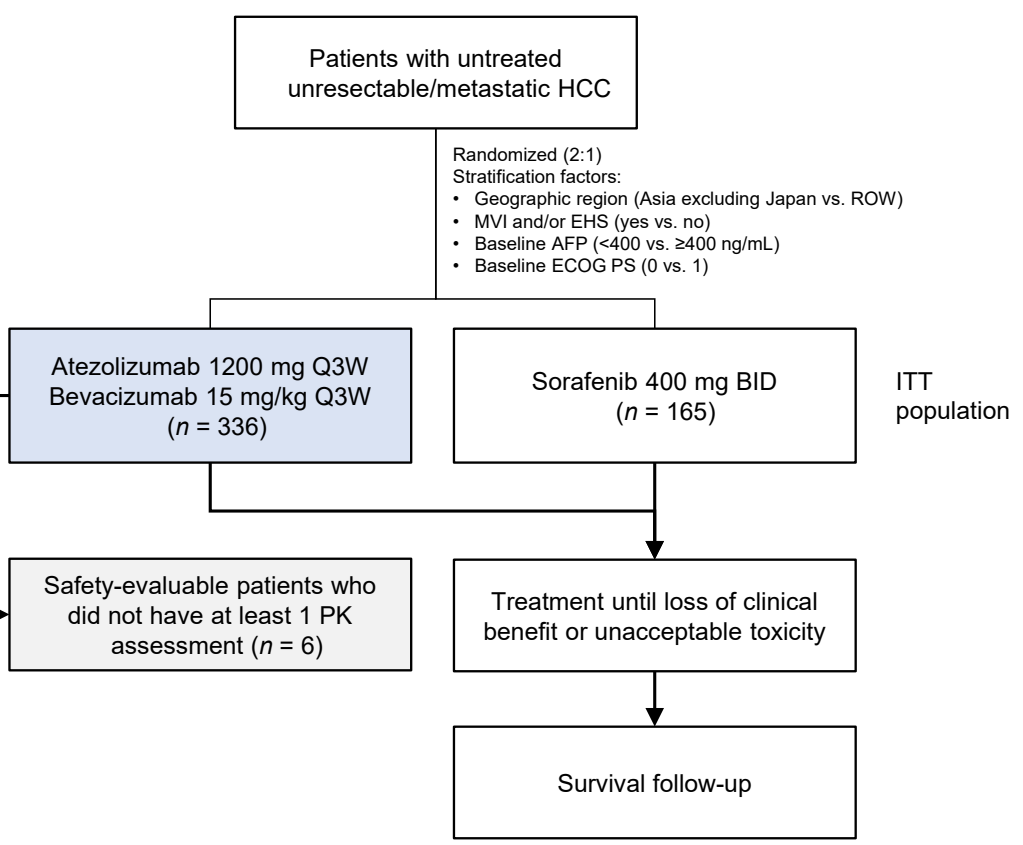

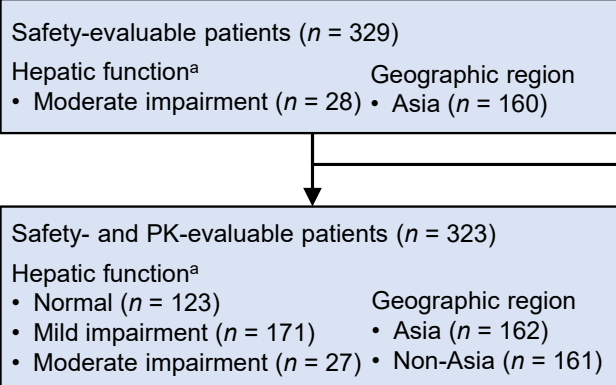

Patients who did not receive at least one full or partial dose of study drug $(n=7)$

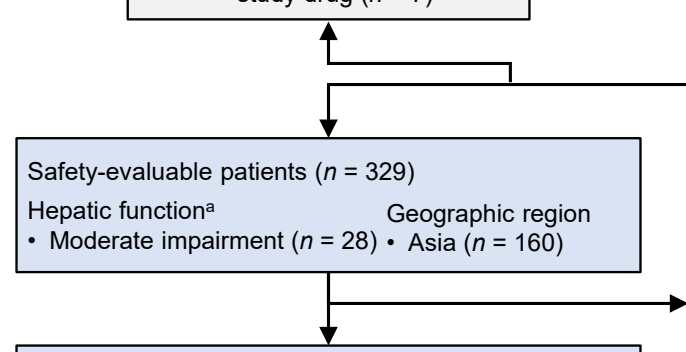

Safety- and PK-evaluable patients $(n=323)$

- Moderate impairment $(n=27) \cdot$ Non-Asia $(n=161)$
MVI and/or EHS (yes vs. no)

Atezolizumab $1200 \mathrm{mg}$ Q3W $(n=336)$

$$
(n=165)
$$

\section{b) $\mathrm{GO} 30140$}

Patients with untreated unresectable/metastatic HCC

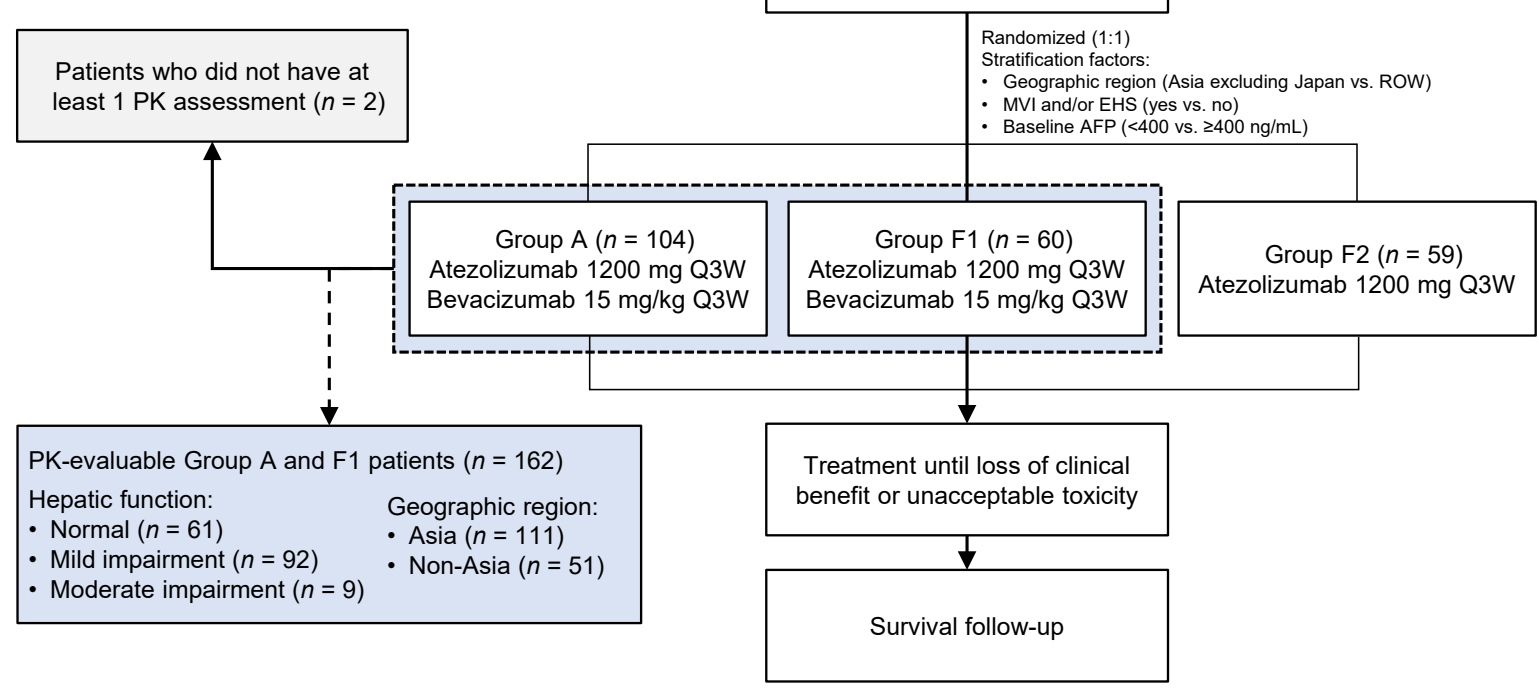

ITT population
Fig. 1. Phase 3 IMbrave150 (a) and phase 1 GO30140 (b) study designs and flowcharts of the analysis populations. The hepatic function refers to that defined by the NCI-ODWG criteria. For purposes of PK and safety assessments, the current analysis includes patients in Japan as part of the Asia subgroup (for geographic region). AFP, $a$-fetoprotein; BID, twice daily; ECOG PS, Eastern Cooperative Oncology Group performance status; EHS, extrahe- patic spread; HCC, hepatocellular carcinoma; ITT, intention to treat; MVI, microvascular invasion; NCI-ODWG, National Cancer Institute Organ Dysfunction Working Group; PK, pharmacokinetic; Q3W, every 3 weeks; ROW, rest of world. ${ }^{\text {a Two patients }}$ with severe hepatic impairment were not included in subgroup analyses.
Atezolizumab + Bevacizumab

Pharmacokinetics and Safety in HCC 
study have been previously reported [10]. Patients were enrolled and randomized 2:1 to atezolizumab 1,200 mg every 3 weeks (Q3W) plus bevacizumab $15 \mathrm{mg} / \mathrm{kg}$ intravenous infusions Q3W (experimental arm: intention to treat [ITT], $n=336$; treated patients, $n=329$ ); or sorafenib $400 \mathrm{mg}$ orally, twice daily, and continuously (comparator arm: ITT, $n=165$; treated, $n=156$ ). Randomization was stratified according to geographic region (Asia [excluding Japan] vs. rest of world [including Japan]), macrovascular invasion or extrahepatic spread or both (presence vs. absence), baseline $\alpha$-fetoprotein (AFP; $<400$ vs. $\geq 400 \mathrm{ng} / \mathrm{mL}$ ), and Eastern Cooperative Oncology Group performance status (0 vs. 1).

Study GO30140 (NCT02715531) is a global, phase 1b, openlabel study evaluating the safety and efficacy of atezolizumab administered with or without bevacizumab in advanced or metastatic solid tumor types, including patients with unresectable HCC who had received no prior systemic therapy. Data from arms A and $F$ have been reported [11]. In group A (ITT, $n=104$ ), patients received atezolizumab 1,200 mg Q3W in combination with bevacizumab $15 \mathrm{mg} / \mathrm{kg}$ Q3W. In group F, patients were randomized 1:1 to either the atezolizumab + bevacizumab combination from group A (group F1 [ITT, $n=60$ ]) or atezolizumab monotherapy (group F2 [ITT, $n=59$ ]). Randomization into group F was stratified based on the region (Asia [excluding Japan] vs. rest of world [including Japan]), baseline AFP levels ( $<400$ vs. $\geq 400 \mathrm{ng}$ per $\mathrm{mL}$ ), and presence or absence of macrovascular invasion, extrahepatic spread, or both.

PK-evaluable patients had at least $1 \mathrm{PK}$ assessment. PK was evaluated as a prespecified exploratory objective in IMbrave150 and as a secondary objective in arms A and F of GO30140. Atezolizumab PK and safety data by hepatic impairment status and world region are from the IMbrave150 population; bevacizumab PK data by hepatic impairment status and world region are from the GO30140 population. The specific world regions are described further below. PK data for bevacizumab in IMbrave150 were not collected, as results had been described in GO30140, while PK data for atezolizumab in GO30140 are not described herein on account of assessment in the large, randomized, phase 3 setting in IMbrave150. Both studies were conducted in accordance with the Declaration of Helsinki and Good Clinical Practice Guidelines, following approval by independent review boards or Ethics Committees at each institution. Written informed consent was obtained from each patient. The analysis populations are depicted in Figure 1.

\section{Hepatic Impairment Classification}

Hepatic impairment status was determined using several criteria, including National Cancer Institute Organ Dysfunction Working Group (NCI-ODWG) criteria, the Child-Pugh score, and the albumin/bilirubin (ALBI) criteria, as described below. The use of NCI-ODWG criteria in PK analyses is supported by an evaluation of study data from 2000 to 2014 US FDA product labels $(n=1,841$ patients in 65 studies) that found NCI-ODWG to be more sensitive than Child-Pugh criteria for classifying changes in exposure [37]. The NCI-ODWG criteria are also generally accepted by global regulatory bodies, are included in the prescribing information for atezolizumab and bevacizumab in patients with HCC $[38,39]$, and have been used to guide dosing decisions for other therapeutic agents as well [40]. The NCI-ODWG criteria are based on bilirubin and aspartate aminotransferase (AST) levels. Subgroup A was defined by bilirubin $\leq$ upper limit of normal (ULN) and AST $\leq$ ULN; subgroup B1 was defined by total bilirubin $\leq \mathrm{ULN}$ and AST $>$ ULN; subgroup B2 was defined as total bilirubin $>1-1.5 \times$ ULN and any AST; subgroup C was defined as bilirubin $>1.5-3.0 \times$ ULN and any AST; and, lastly, subgroup D was defined by total bilirubin $>3 \times$ ULN and any AST. The ULN for bilirubin and AST were set as 17.1 $\mu \mathrm{mol} / \mathrm{L}$ and $40 \mathrm{U} / \mathrm{L}$, respectively. NCI-ODWG groups were categorized as: A (normal hepatic function), B1 and B2 (mild hepatic impairment), $\mathrm{C}$ (moderate hepatic impairment), and $\mathrm{D}$ (severe hepatic impairment). In addition to NCI-ODWG classification, Child-Pugh scores were calculated based on 5 clinical and laboratory criteria, with points assigned to each criterion based on increasing severity. For encephalopathy: 1 point (none), 2 points (grades 1 and 2), 3 points (grades 3 and 4); ascites: 1 point (none), 2 points (slight), and 3 points (moderate); bilirubin: 1 point $(<2$ $\mathrm{mg} / \mathrm{mL}), 2$ points $(2-3 \mathrm{mg} / \mathrm{mL})$, and 3 points $(>3 \mathrm{mg} / \mathrm{mL})$; albumin: 1 point $(>3.5 \mathrm{mg} / \mathrm{mL}), 2$ points $(2.8-3.5 \mathrm{mg} / \mathrm{mL})$, and 3 points $(<2.8 \mathrm{mg} / \mathrm{mL})$; prothrombin time (seconds prolonged): 1 point $(<4$ s), 2 points ( $4-6 \mathrm{~s}$ ), and 3 points ( $>6 \mathrm{~s}$ ), with an international normalized ratio (INR) used as a substitute for prothrombin time: 1 point (INR <1.7), 2 points (INR 1.7-2.2), and 3 points (INR > 2.2). Patients were categorized into groups as A (5-6 points), B (7-9 points), or $\mathrm{C}$ (10-15 points) [41]. The mild, moderate, and severe NCI-ODWG groups correspond to Child-Pugh scores of 6, 7-9, and $10-12$, respectively [42]. Finally, the liver function was also assessed based on ALBI levels (the ALBI score) [43]. The ALBI grade was calculated by means of the formula $0.66 \times \log _{10}$ (total bilirubin $[\mu \mathrm{mol} / \mathrm{L}])-0.085$ (albumin $[\mathrm{g} / \mathrm{L}]$ ) with the ALBI score stratified as grade $1(-2.60$ or less), grade $2(-2.59$ to -1.39$)$, or grade 3 (greater than -1.39 ). PK analyses based on hepatic impairment were conducted in line with guidance documents from the US FDA and the European Medicines Agency [17, 44].

\section{Geographic Regional Assessments}

Patients in IMbrave150 were retrospectively grouped into the following geographic regions: Asia (mainland China, Hong Kong, Japan, Singapore, South Korea, and Taiwan) and non-Asia (Australia, Canada, Czech Republic, France, Germany, Italy, Poland, Russia, Spain, the UK, and the USA). The bevacizumab PK data set for GO30140 included patients in Asia (Japan, Korea, and Taiwan) and non-Asia (Australia, New Zealand, and the USA). An additional subgroup analysis evaluated PK data specifically in patients enrolled in Japan versus those enrolled outside of Japan, for atezolizumab and bevacizumab, as described above.

\section{PK Sampling and Bioanalytical Methods}

Serum samples for measuring atezolizumab concentrations were collected at cycle 1 (30 min after infusion) and cycles 2, 3, 4, 8 , and 12 (before dose). Serum samples for measuring bevacizumab concentrations were collected at cycles 1 and 3 (30 min after infusion) and cycle 3 (before dose). Atezolizumab and bevacizumab concentrations were quantified by ELISA, with 60- and 78$\mathrm{ng} / \mathrm{mL}$ lower limits of quantification in human serum, respectively. Blood samples were centrifuged at 1,500-2,000 $\mathrm{g}$ for $15 \mathrm{~min}$ at $4^{\circ} \mathrm{C}$. The serum samples were then stored at $-60^{\circ} \mathrm{C}$ or lower. The methodology for serum concentration measurements was validated: (1) for atezolizumab, inter-run and intra-run precision values (reported as \%coefficient of variation) were $\leq 4.59$ and $\leq 4.12 \%$, respectively, and inter-run and intra-run accuracy (reported as percent relative error) was -7.13 to $4.17 \%$, and -7.17 to $3.96 \%$, respectively; (2) for bevacizumab, inter-assay precision ranging from 11 
to $15 \%$, intra-assay precision ranged from 9 to $12 \%$, and the mean accuracy (\% recovery of analyte added to matrix) ranged from 84 to $125 \%$. The assays specifically detected analytes in disease stage samples. No interference from either assay was observed from hemolysis, lipemia, and comedications.

\section{PopPK Model}

A previously developed 2-compartment popPK model of atezolizumab based on phase 1 data [12] was used in the PK analyses. According to the phase 1 popPK model, the typical clearance (CL, in $\mathrm{L} /$ day) of atezolizumab for patient $i$ was

$$
\mathrm{CL}_{i}=\left[0.200 \times\left(\frac{\mathrm{ALBU}_{i}}{40}\right)^{-1.12} \times\left(\frac{\mathrm{BWT}_{i}}{77}\right)^{0.008} \times\left(\frac{\text { Tumor burden }_{i}}{63}\right)^{0.125}\right] \times(1.159 \text { if ADA is positive }),
$$

where BWT is body weight in $\mathrm{kg}$; ALBU is albumin in $\mathrm{g} / \mathrm{L}$; and tumor burden is expressed in $\mathrm{mm}$; antidrug antibodies (ADAs) is treatment-emergent status of ADAs: post-baseline ADA is negative when post-dose samples were collected after baseline or the ADA signal was not enhanced after baseline (treatment unaffected); post-baseline ADA is positive when treatment induced or treatment enhanced; and post-baseline ADA is missing when all post-dose samples were missing. The typical volumes in L of distribution of the central compartment $\left(V_{1}\right)$ and the peripheral compartment $\left(V_{2}\right)$ of atezolizumab for patient $i$ were

$$
\begin{aligned}
& V_{1_{i}}=\left[3.28 \times\left(\frac{\mathrm{BWT}_{i}}{77}\right)^{0.559} \times\left(\frac{\mathrm{ALBU}_{i}}{40}\right)^{-0.350}\right] \times(0.871 \text { if female }) . \\
& V_{2 i}=3.63 \times(0.728 \text { if female })
\end{aligned}
$$

A combined model with proportional and additive components described the residual error. The PK sampling schedule in the phase 1 study (from which the popPK model was developed [12]) was more intensive than in the phase 3 study, from which mostly trough PK samples were collected. Therefore, the popPK model was not redeveloped and the parameters were not re-estimated, and IMbrave150 data served as external validation to evaluate the performance of the model as previously done in a metastatic urothelial carcinoma study [12]. The popPK analysis was performed with NONMEM v7.4 (ICON Development Solutions, Ellicott City, MD, USA) in conjunction with Perl-Speak-NONMEM (v3.7.6; Uppsala University, Uppsala, Sweden). Covariate effects related to liver function, that is, AST, alanine aminotransferase (ALT), and bilirubin in the HCC population, were tested. When covariate values in the popPK data set were missing in $<15 \%$ of the total number of patients, the values were imputed to median values for continuous covariates or to the most frequent category for categorical covariates. Performance of the phase 1 popPK model on the current data set was evaluated by goodness-of-fit plots and prediction-corrected visual predictive check [45]. The exposure metrics for atezolizumab were derived using the individual PK parameters estimated with the phase 1 popPK model (post hoc option with maxeval $=0$ ).

\section{$P K$ and Statistical Analysis}

Descriptive statistics of observed PK parameters (atezolizumab and bevacizumab serum peak concentration $\left[C_{\max }\right]$ and trough/ pre-dose concentration $\left.\left[C_{\min }\right]\right)$ were calculated using Phoenix WinNonlin version 6 or higher (Pharsight, Inc., Mountain View, CA, USA). For each value, the geometric mean and \% geometric coefficients of variation (\%geo CV) were determined. These data were summarized in tabulated form, as well as using box plot analysis by nominal time for direct comparison. The impact of mild or moderate hepatic impairment on either atezolizumab or bevacizumab PK was assessed by comparing exposures with those from patients with normal hepatic function after a single dose and at steady state. To evaluate potential differences by region, we compared the PK in patients enrolled in Asia (including Japan) with that in patients in non-Asian regions. The popPK model was used to derive the area under the concentration-time curve (AUC), $C_{\max }$, and $C_{\min }$ in cycle 1 and beyond, including at steady state. Geometric means and \%geo CV were computed and tabulated, as well as the geometric mean ratio (GMR) to the reference category and its $90 \%$ CI. No formal statistical testing was performed when making comparisons, given that the clinical trials were not powered for the purpose of assessing the potential impact of hepatic impairment or geographic region.

\section{Safety Assessments}

In IMbrave150, the analysis population for safety, a secondary endpoint in the study, consisted of randomized patients who received at least 1 full or partial dose of study treatment. Safety assessments included the incidence, nature, and severity of adverse events (AEs) and laboratory abnormalities graded per the National Cancer Institute Common Terminology Criteria for Adverse Events, Version 4.0 (NCI-CTCAE v4.0). Laboratory safety assessments included the regular monitoring of hematology and blood chemistry. The following events occurring during or after the first dose of study treatment were summarized by treatment group and NCI-CTCAE grade: all AEs, all serious AEs (SAEs), all severe AEs (grade $\geq 3$ ), and all AEs leading to study drug discontinuation or interruption. Multiple occurrences of the same event were counted once at the maximal severity.

\section{Results}

\section{Study Population}

The IMbrave $150 \mathrm{PK}$ data set comprised 323 patients in the atezolizumab + bevacizumab arm with at least $1 \mathrm{PK}$ assessment, of whom 123 (38\%), 171 (53\%), 27 (8.4\%), and $2(0.6 \%)$ had normal hepatic function or mild, moderate, or severe hepatic impairment, respectively, at baseline by NCI-ODWG criteria. The distribution of hepatic impairment categorized by Child-Pugh and ALBI classification, and their respective overlap with NCI-ODWG classification, is shown in online suppl. Table 1 (see www. karger.com/doi/10.1159/000515817 for all online suppl. material). All 162 IMbrave150 patients enrolled in Asian regions (including Japan) were Asian, and of the 161 patients enrolled outside of Asia, 20 (12.4\%) were Asian. Patient characteristics for IMbrave 150 are shown in Table 1 and were generally balanced between subgroups. The patient population was predominantly male (ranging from 80 to $85 \%$ ), and the median age ranged from 62 to 66 years 
Table 1. Baseline demographic and clinical characteristics for patients from IMbrave150 (current study analysis populations)

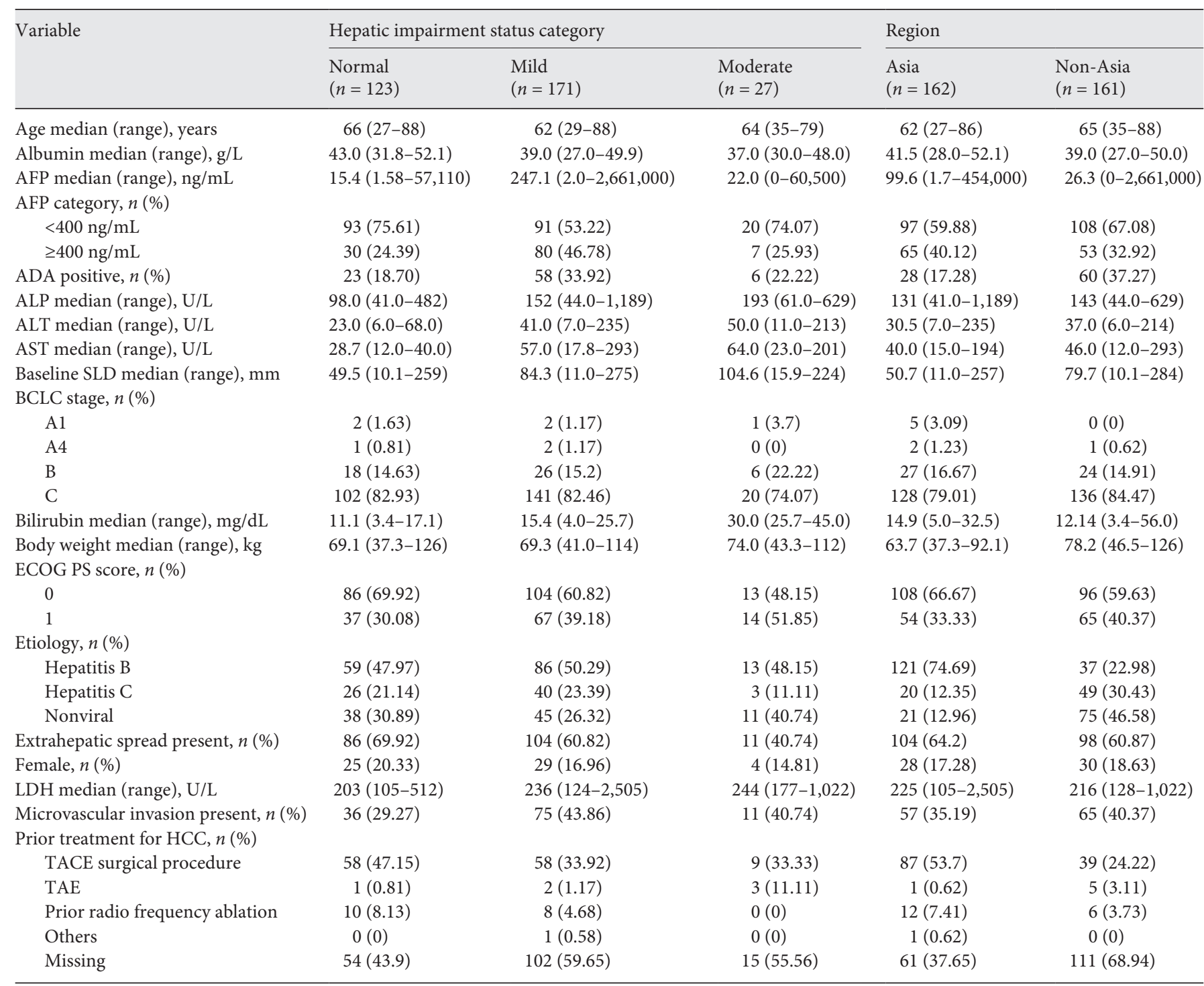

Hepatic impairment is based on the NCI-ODWG criteria scale (normal function, bilirubin $\leq$ ULN and AST $\leq$ ULN; mild impairment, bilirubin $\leq$ ULN and AST $>$ ULN, or bilirubin $>1-1.5 \times$ ULN and AST any; moderate impairment, bilirubin $>1.5-3.0 \times$ ULN and AST any). The ULN for bilirubin was set as $17.1 \mu \mathrm{mol} / \mathrm{L}$ and $40 \mathrm{U} / \mathrm{L}$ for AST. Two patients with severe hepatic impairment were not included in hepatic impairment status categories. Patients were grouped into 2 geographic regions: inside Asia: China, Hong Kong, Japan, South Korea, and Taiwan; and outside Asia: Australia, Canada, Czech Republic, France, Germany, Italy, Poland, Russia, Spain, the UK, and the USA. Nonviral causes include alcohol, other, and unknown non-hepatitis B and C causes. The BCLC staging system ranks HCC in 5 stages, beginning at 0 (very early stage) and progressing from A (early stage) to D (terminal stage). ECOG PS scores range from 0 to 5, with higher numbers reflecting greater disability. ADA, antidrug antibody; AFP, a-fetoprotein; ALP, alkaline phosphatase; ALT, alanine aminotransferase; AST, aspartate aminotransferase; BCLC, Barcelona Clinic Liver Cancer; ECOG, European Cooperative Oncology Group performance status; HCC, hepatocellular carcinoma; LDH, lactate dehydrogenase; NCI-ODWG, National Cancer Institute Organ Dysfunction Working Group; SLD, sum of longest diameters; TACE, trans-arterial chemoembolization; TAE, trans-arterial embolization; ULN, upper limit of normal.

across subgroups. In all groups apart from the moderate hepatic impairment subgroup, the majority of patients had an Eastern Cooperative Oncology Group performance status of 0 . The median body weight was $23 \%$ lower for patients in Asia $(n=162)$ than outside of Asia $(n=$
161). The degree of hepatic impairment per NCI-ODWG observed across regions in IMbrave 150 was comparable (79 [52\%] and 12 [8\%] of patients from the Asia subgroup and 93 [53\%] and 15 [9\%] from the non-Asia subgroup had mild and moderate hepatic impairment, respectively). 
Table 2. popPK summary statistics, the geometric mean (\%geo CV) of atezolizumab exposure metrics by hepatic function, and the geographic region for patients in IMbrave 150

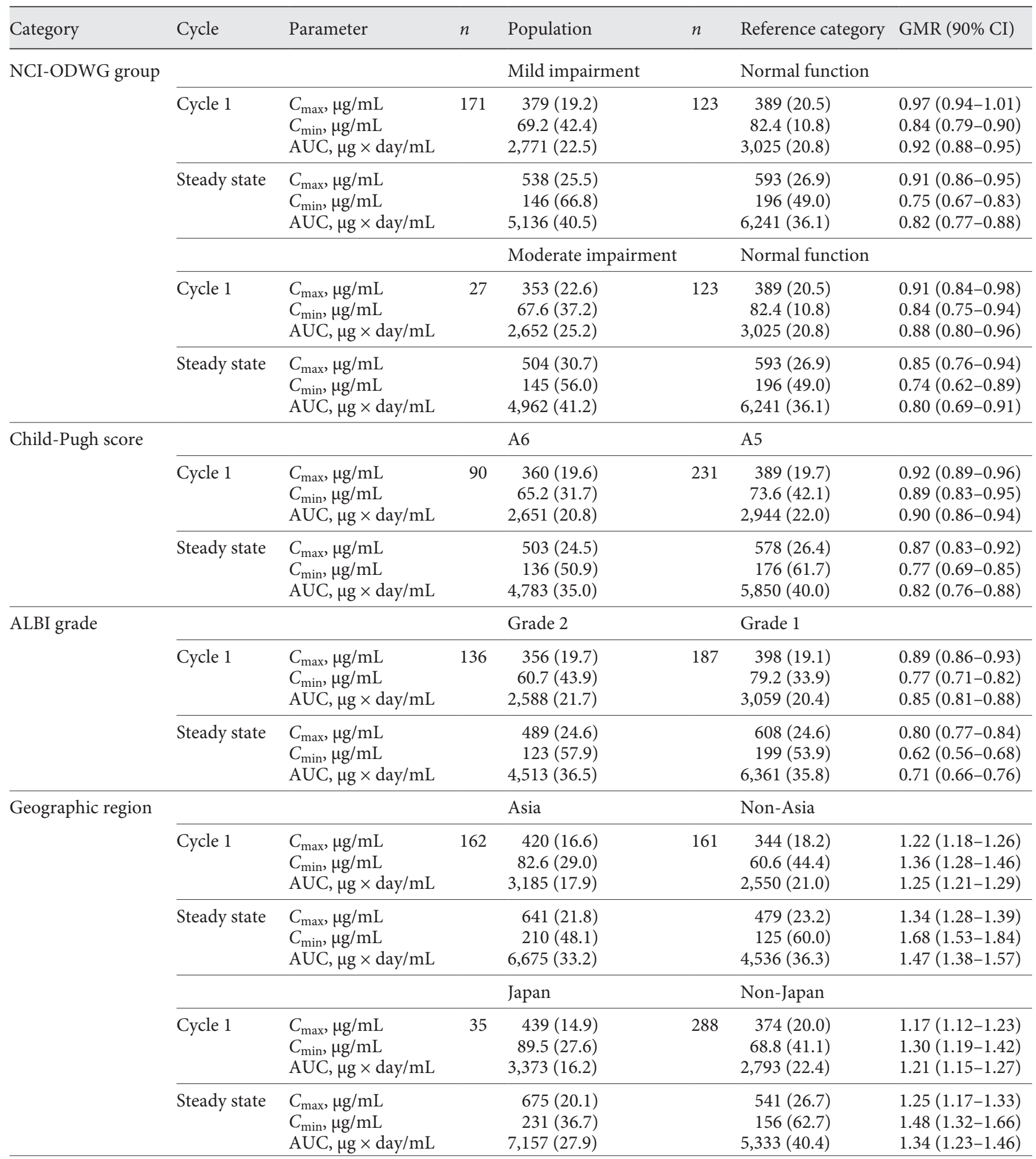

Atezolizumab + Bevacizumab

Pharmacokinetics and Safety in HCC 
All patients ( $n=123$ with normal hepatic function, $n=171$ and $n=27$ with mild and moderate hepatic impairment; $n=162$ and $n=161$ patients enrolled in Asia and non-Asian regions) in IMbrave150 who received atezolizumab 1,200 mg in combination with 15 $\mathrm{mg} / \mathrm{kg}$ of bevacizumab Q3W. All values are geometric mean (\%geo CV), except for GMR. The GMR with respect to the reference category and its $90 \%$ CI is shown. Summary statistics for patients with severe hepatic impairment were not conducted due to the small sample size $(n=2)$. Patients with unknown Child-Pugh score were not included in the summary $(n=2)$. "Asia” geographic region includes Japan. AUC, area under the concentration-time curve; ALBI, albumin/bilirubin; $C_{\max }$, maximum concentration; $C_{\text {min }}$, minimum concentration; geo CV, geometric coefficient of variation; GMR, geometric mean ratio; NCI-ODWG, National Cancer Institute Organ Dysfunction Working Group; PK, pharmacokinetics; popPK, population PK; Q3W, every 3 weeks.

In the IMbrave150 population, median levels of liver function enzymes (e.g., AST, alkaline phosphatase, and ALT) and median baseline tumor burden (sum of the longest diameters) appeared to be greater in patients with increased hepatic dysfunction, assessed either by NCIODWG criteria (shown in Table 1) or by Child-Pugh or ALBI criteria (shown in online suppl. Table 2). The percentage of patients with AFP $>400 \mathrm{ng} / \mathrm{mL}$ was similar among patients in Asia and patients outside of Asia. The majority of patients across all subgroups had Barcelona Clinic Liver Cancer stage C liver cancer, consistent with the typical late-stage presentation of HCC [46]. For HCC disease characteristics, nonviral etiology or hepatitis $\mathrm{C}$ were more common outside of Asia, while hepatitis B was most common in Asia. Extrahepatic spread was present in the majority of patients, apart from those with moderate hepatic impairment. Overall, the baseline demographic and disease characteristics were reflective of an unresectable HCC population with varying degrees of hepatic impairment and the diverse geographic regional origin.

In pooled treatment, arms A and F1 from the GO30140 phase $1 \mathrm{~b}$ study, 61,92 , and 9 patients were classified as having normal hepatic function, mild hepatic impairment, and moderatehepatic impairment per NCI-ODWG criteria, respectively; 111 of these patients were enrolled in Asia, and the remaining 51 patients were enrolled outside of Asia. In GO30140, 111 patients enrolled in Asian regions, and all were Asian; 7 of the 51 patients (13.7\%) who enrolled outside of Asia were Asian. Overall, across both studies, the percentage of Asian patients enrolled outside of Asia was limited to $12.7 \%$.

\section{PK in Hepatic Impairment Groups}

Performance of the phase 1 popPK model by the method of external validation is shown in online suppl. Figure 1. A summary of popPK-derived atezolizumab PK data by visit for each hepatic function group in IMbrave150 is shown in Table 2, while observed PK is shown in Figure 2a and online suppl. Figure $2 \mathrm{a}$ and $\mathrm{b}$. A summary of observed bevacizumab PK data by visit across hepatic impairment groups in GO30140 is shown in Table 3 and Figure 2c and online suppl. Figure $2 \mathrm{~d}$ and e. Distributions shown in Figure $2 \mathrm{a}$ and $\mathrm{c}$ and in online suppl. Figure $2 \mathrm{a}, \mathrm{b}, \mathrm{d}$, and e show that there was considerable overlap in exposure across hepatic impairment groups for both atezolizumab and bevacizumab concentrations. The calculated GMR for atezolizumab AUC (cycle 1 and steady state) also showed high similarity across hepatic function subgroups, ranging from 0.82 to 0.92 for patients with mild hepatic impairment (NCI-ODWG criteria) and $0.80-0.88$ for those with moderate impairment, relative to patients with normal hepatic function; the range was $0.82-0.90$ for Child-Pugh A6 versus A5 and 0.71-0.85 for ALBI grade 2 versus 1 (Table 2). GMRs for peak and trough concentrations of bevacizumab within cycle 1 and cycle 3 ranged from 0.94 to 97 and 0.94 to 1.27 , respectively, for patients with mild or moderate hepatic impairment (NCI-ODWG criteria) compared with patients with normal hepatic function; the ranges were $0.89-1.03$ for Child-Pugh A6 versus A5 and 0.86-0.92 for ALBI grade 2 versus 1 (Table 3 ). In a post hoc analysis evaluating the NCI-ODWG, Child-Pugh, and ALBI-defined subgroups, $\mathrm{PK}$ parameters between the groups were not found to be different (exploratory $p \leq 0.01$ ). Online suppl. Figures $3 \mathrm{a}, 4 \mathrm{a}$, and $\mathrm{b}$ shows interindividual $\mathrm{PK}$ variability trends by hepatic function. Interindividual variability relationships between random effects of central clearance, central volume of distribution, and peripheral volume of distribution in the popPK model using categorical variables of hepatic function indicated no meaningful effect on the popPK parameters.

\section{PK by Geographic Region}

The observed atezolizumab $\mathrm{PK}$ data by region are shown in Figure 2b and online suppl. 2c; and popPK-derived metrics are shown in Table 2; data for bevacizumab are shown in Figure $2 \mathrm{~d}$ and online suppl. $2 \mathrm{f}$ and Table 3. The larger differences in GMR (increases of 0.36) for cycle $1 C_{\min }$ of atezolizumab between patients in Asia versus non-Asia are 


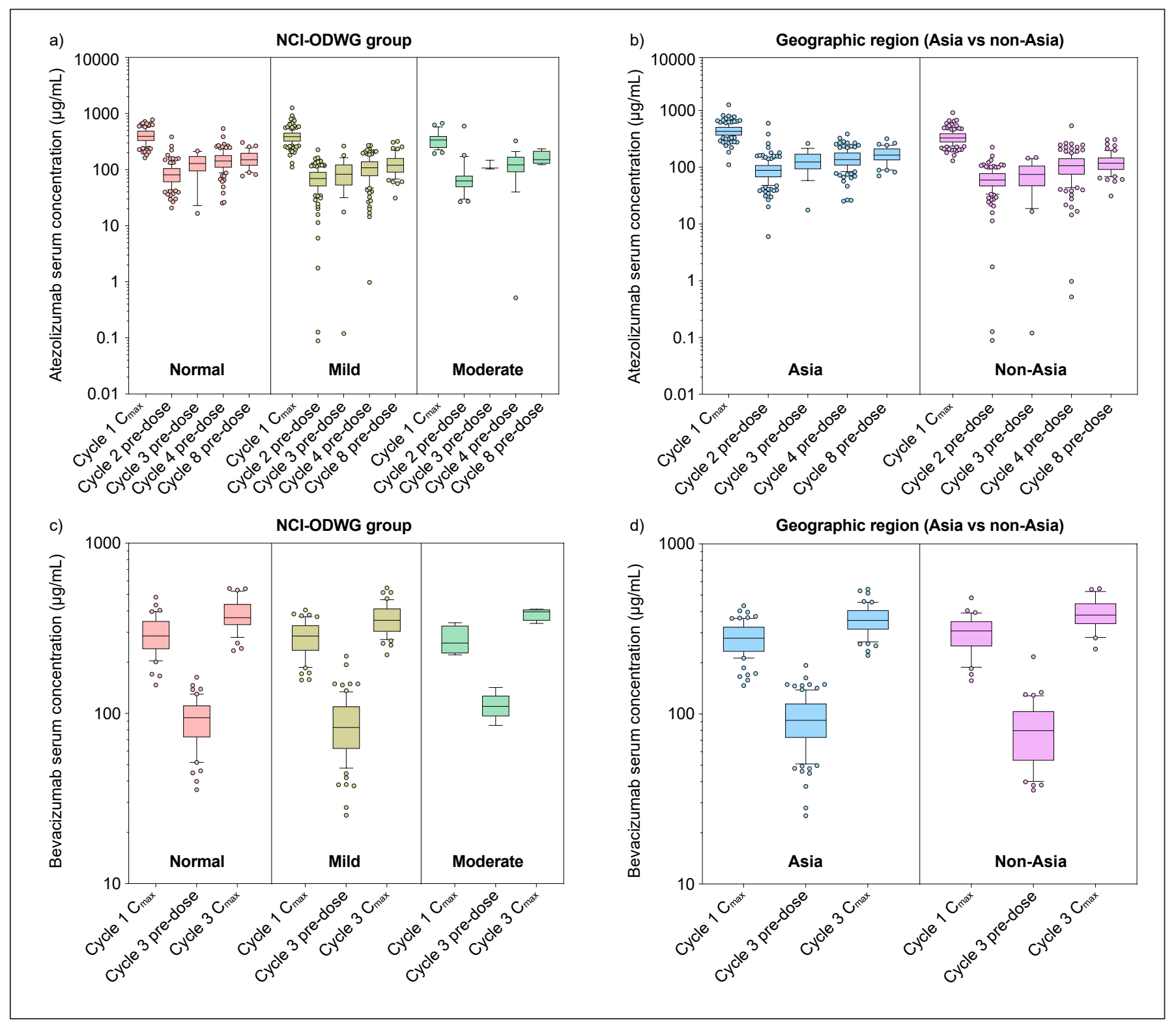

Fig. 2. PK of atezolizumab and bevacizumab by hepatic function (a, c) and geographic region (b, d) following single and multiple administrations of atezolizumab $1,200 \mathrm{mg}$ with bevacizumab 15 $\mathrm{mg} / \mathrm{kg}$ Q3W in patients with HCC. Box plots include the median, 25th, and 75th percentiles for values at indicated visits, and whiskers refer to 10th and 90th percentiles, with outliers (open circles) plotted as single points vertically. $C_{\max }$, maximum concentration; HCC, hepatocellular carcinoma; PK, pharmacokinetics; Q3W, every 3 weeks. within the interindividual variability (up to 0.44 ) observed. As shown in Figure $2 \mathrm{~b}$ and $\mathrm{d}$, there was also considerable overlap in both atezolizumab and bevacizumab concentrations across both geographic regions, evident in the broad confidence intervals. The calculated GMR shows similarities for $C_{\max }, C_{\min }$, and AUC for atezolizumab across the 2 regions and high similarity for bevacizumab between patients in and outside Asia. For instance, atezolizumab AUC GMRs (cycle 1 and steady state) for patients in Asia (vs. outside Asia) had ranged from 1.25 to 1.47. GMRs for peak and trough concentrations of bevacizumab within cycle 1 and cycle 3 ranged from 0.94 to 1.15 for patients enrolled in Asia versus outside Asia. The largest difference in cycle 1 atezolizumab exposure across regions was in $C_{\min }$, which increased by 0.36 in patients in Asian regions compared with patients in non-Asian regions. For exploratory purposes, when normalized for dose by body weight, the range of GMRs of observed exposure of atezolizumab across mul- 
Table 3. Observed PK summary statistics, the geometric mean (\%geo CV) of bevacizumab exposure metrics by hepatic function, and the geographic region for patients in GO30140

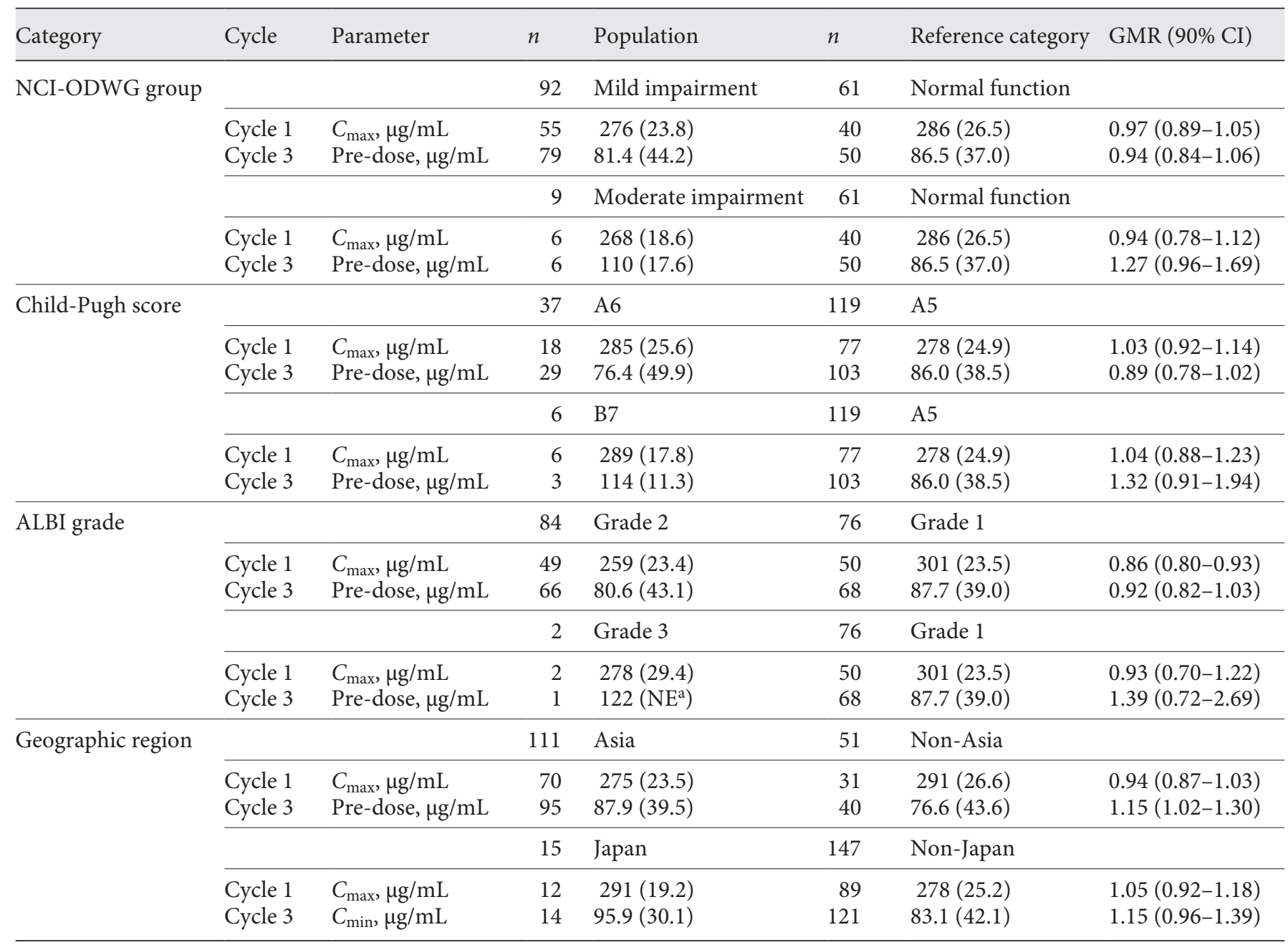

All values are geometric mean (\%geo CV), except for GMR. The GMR with respect to the reference category and its $90 \%$ CI are shown. Patients with an unknown Child-Pugh score were not included in the summary $(n=2)$. "Asia” geographic region includes Japan. Patient numbers are shown next to subgroup headings, and PK sample numbers are shown next to sampling time points. ALBI, albumin/bilirubin; $C_{\max }$, maximum concentration; $C_{\min }$, minimum concentration; geo $\mathrm{CV}$, geometric coefficient of variation; GMR, geometric mean ratio; NCI-ODWG, National Cancer Institute Organ Dysfunction Working Group; PK, pharmacokinetics. ${ }^{\text {a }}$ NE refers to not evaluable based on $n=1$.

tiple cycles after the first dose were $0.19-0.38$ closer between groups, confirming that subtle differences were related to body weight. Further, ethnicity (race/region) was also explored as a covariate on the atezolizumab popPK model and found not to have an effect (at the exploratory $p$ value of 0.01). Additional analyses of interindividual PK variability trends by region are shown in online suppl. Figures $3 b$ and $4 c$, while those for viral etiology are shown in online suppl. Figure $3 \mathrm{c}$. Interindividual variability relationships between random effects of central clearance, central volume of distribution, and peripheral volume of distribution in the popPK model using categorical variables of region and etiology indicated no meaningful effect on the popPK parameters. Finally, as Japanese patients may have HCC tumors with etiology distinct from other Asian patients, an additional analysis of patients enrolled in Japan versus outside Japan showed that the magnitude of GMR percent differences for evaluated metrics for atezolizumab and bevacizumab were in line with the Asia versus nonAsia comparison (shown in Tables 2, 3). 
Table 4. Summary of AEs in IMbrave150 (atezolizumab + bevacizumab)

\begin{tabular}{|c|c|c|c|}
\hline & $\begin{array}{l}\text { Moderate hepatic } \\
\text { impairment }(n=28)\end{array}$ & $\begin{array}{l}\text { Asia } \\
(n=166)\end{array}$ & $\begin{array}{l}\text { Overall population } \\
(n=329)\end{array}$ \\
\hline Total number of patients with at least $1 \mathrm{AE}$ & $27(96.4)$ & $163(98.2)$ & $323(98.2)$ \\
\hline Grade $3 / 4 \mathrm{AE}$ & $16(57.1)$ & $94(56.6)$ & $186(56.5)$ \\
\hline Treatment-related grade $3 / 4 \mathrm{AE}$ & $9(32.1)$ & $66(39.8)$ & $117(35.6)$ \\
\hline Grade $5 \mathrm{AE}$ & $2(7.1)$ & $5(3.0)$ & $15(4.6)$ \\
\hline SAE & $14(50.0)$ & $54(32.5)$ & $125(38.0)$ \\
\hline Treatment-related SAE & $6(21.4)$ & $28(16.9)$ & $56(17.0)$ \\
\hline AE leading to withdrawal from any study treatment & $5(17.9)$ & $18(10.8)$ & $51(15.5)$ \\
\hline AE leading to dose modification or interruption of any study treatment & $14(50.0)$ & $72(43.4)$ & $163(49.5)$ \\
\hline
\end{tabular}

Values are presented as $n(\%)$. Includes AEs with the onset date on or after the date of the first dose of study drug. Investigator text for AEs is encoded using Medical Dictionary for Regulatory Activities version 22.0. Percentages are based on $n$ in the column headings. Multiple occurrences of the same AE in one individual are counted only once. Treatment-related grade 3/4 AE refers to highest grade experienced. AE, adverse event; $\mathrm{SAE}$, serious $\mathrm{AE}$.

Safety by Hepatic Impairment and Geographic Region

A safety summary is shown in Table 4 . The AE frequency was similar in patients with moderate hepatic impairment (96.4\%) and those in Asia (98.2\%) and in the overall population (98.2\%). Similar observations for the following AEs were seen in patients with moderate hepatic impairment and the Asian global region, respectively, versus the overall population: (1) all-cause grade $3 / 4$ AEs (57.1\% and $56.6 \%$ vs. $56.5 \%)$, (2) treatment-related grade $3 / 4$ AEs ( $32.1 \%$ and $39.8 \%$ vs. $35.6 \%$ ), and (3) treatment-related SAEs $(21.4 \%$ and $16.9 \%$ vs. $17.0 \%)$ across respective groups. All-cause and treatment-related grade 5 AEs occurred at low frequency across groups (3.0-7.1\% and $0-2.4 \%$, respectively). The number of AEs leading to withdrawal from study treatment was similar in patients with moderate hepatic impairment (17.9\%) and the overall population (15.5\%). Some numerical differences were observed in rates of SAEs $(50.0 \%$ in patients with moderate hepatic impairment vs. $38.0 \%$ overall) and AEs leading to dose interruption of any study treatment $(43.4 \%$ in the Asia subgroup vs. $49.5 \%$ overall).

\section{Discussion}

In this analysis of patients with HCC from the IMbrave150 and GO30140 studies, we performed comprehensive PK and safety analyses to evaluate atezolizumab and bevacizumab in patient subsets defined by hepatic impairment status and geographic region. We found that neither hepatic impairment nor the geographic region had a clinically meaningful impact on PK or safety. The lack of effect of hepatic impairment on PK was supported using several classification systems for defining impairment. Our data suggest that no dose adjustments to atezolizumab plus bevacizumab in HCC are needed in these populations.

Although $\mathrm{mAbs}$ are cleared by ubiquitous nonspecific proteolytic catabolism by lysosomal degradation to amino acids [18], the liver may have other roles in catabolism that could potentially alter the PK of administered mAbs. Neonatal Fc receptor binding, target-mediated drug disposition, $\mathrm{Fc} \gamma$ receptor binding, and other mechanisms may further alter exposure [47-51]. Hepatic impairment, in addition to differing disease stages and variability in Fc $\gamma$ expression levels, may lead to decreased Fc $\gamma$ R expression, decreased $\mathrm{F} c \gamma \mathrm{R}$ functionality, or both and consequently alter clearance and exposure [49]. In this study, 53 and $8 \%$ of patients in IMbrave 150 (and 56 and 6\% in GO30140) were classified as having mild or moderate hepatic dysfunction at baseline, respectively, by NCIODWG criteria. This observation is consistent with knowledge that patients with HCC generally present at an advanced disease stage [46]. However, despite the high rates of hepatic impairment seen in these studies, no clinically relevant impact on PK was observed for atezolizumab or bevacizumab. It should be noted however that the impact of hepatic impairment on atezolizumab clearance may also be confounded by associated poor disease status, including factors like low albumin, cachexia, or others 
known to impact checkpoint inhibitor clearance [52]. These are the first data on the impact of hepatic impairment on the PK of atezolizumab and bevacizumab in HCC; indeed, despite the established nature of bevacizumab, the PK implications of hepatic impairment by NCI-ODWG criteria were previously unknown and had not been investigated in any tumor type $[16,53]$. Importantly, we also demonstrated that safety data in this study were generally comparable for patients with moderate hepatic impairment per NCI-ODWG criteria versus the overall population. Lastly, recent data in the literature support the use of the NCI-ODWG classification system for hepatic impairment. A contemporary survey of 1,336 oncology clinical trials found that among trials with definitions for liver function thresholds, a combination of bilirubin and transaminase levels were routinely used in $33-64 \%$ of studies [54]. Further, in a 2019 study of a single company's drug portfolio, NCI-ODWG criteria were found to be used in $22(69 \%)$ of 32 hepatic impairment studies for the portfolio's FDA-approved oncology drugs [55].

Regional and ethnic factors having the potential to influence PK, and their impact on the extrapolation of clinical data, can be extrinsic (environmental and cultural, e.g., food diet and concomitant medications) or intrinsic (e.g., genetic polymorphisms). Limited data on ethnic sensitivity of the PK of mAbs in patients with HCC have been available, although $\mathrm{mAbs}$ are likely to be less sensitive to ethnic factors than small molecules $[49,50]$. In our analysis, atezolizumab showed marginal PK differences between groups defined by the geographic region, likely due to flat dosing and the smaller body weight of patients in the IMbrave150 Asian population (approx. 23\% lower for patients in Asia vs. outside of Asia). Further, a smaller volume of distribution is expected in Asian versus nonAsian patients. The range of distributions seen with atezolizumab also achieved target saturation, with the lowest cycle 1 geometric mean $C_{\min }$ of $60.6 \mu \mathrm{g} / \mathrm{mL}$ - which was $>10$-fold higher than the target exposure threshold - observed in all subgroups [56]. It should be noted that exposure metrics within the first treatment cycle are more appropriate for comparative purposes, rather than those at steady state, to isolate potentially confounding factors on exposure such as time-varying clearance $[52,57]$. We evaluated the relationships between observations and simulated PK around the atezolizumab GMR for $C_{\max }$, $C_{\text {min }}$, and AUC in cycle 1. Only subtle differences were observed in GMRs across patients in Asia versus outside of Asia, which were mostly related to body weight. Differences in exposure (the largest of which included a differ- ence in GMR of up to 0.36 for atezolizumab $C_{\min }$ in cycle 1) were not clinically meaningful given the large safety margin, the lack of dose-limiting toxicity up to $20 \mathrm{mg} / \mathrm{kg}$ of atezolizumab in the phase la study [12], and the flat exposure-efficacy and exposure-safety relationships [1215] reported for atezolizumab. Results were comparable when the region was limited to patients in Japan only. In this study, the treatment was also safe and well tolerated in all patients, with no consistent observations of more AEs in patients in Asia than in the overall population.

IMbrave150 data demonstrated that the covariate effects for HCC were consistent with those estimated based on phase 1 data in other indications. Specifically, no covariate effect of laboratory values related to liver function (i.e., ALT, AST, and bilirubin) was detected. Prognostic factors commonly associated with HCC were also investigated for their impact on PK of atezolizumab. No consistent trend in interindividual variability in popPK parameters was observed based on hepatic impairment groups. Additionally, liver function categorization based on liver cancer classification Barcelona Clinic Liver Cancer staging system (B or C) did not exhibit significant covariate effects on the PK of atezolizumab. Finally, there does not appear to be an association between etiology, macrovascular invasion, extrahepatic spread, alcohol use, or the AFP level $(<400$ or $\geq 400 \mathrm{ng} / \mathrm{mL})$ and atezolizumab PK. Cycle 1 exposure metrics of atezolizumab were also similar to those estimated in other studies using atezolizumab Q3W monotherapy or in combination with bevacizumab in renal cell carcinoma (IMmotion151 [58]) and with carboplatin and paclitaxel with or without bevacizumab in first-line non-small-cell lung cancer (IMpower150 [59]). Differences in body weight, a covariate for clearance of mAbs, were observed between Asian and non-Asian subgroups. Across global regions, bevacizumab dosing is based on body weight, and atezolizumab uses flat dosing that does not require adjustments to account for regional body size differences. The similarity in dosing regimens for Western versus Asian regions is also seen with other mAbs; a survey of 22 biologics (predominantly $\mathrm{mAbs}$ ) for oncology use approved in Japan, China, and the USA prior to 2018 showed no differences between dosing regimens used in Asian and Western populations [60].

The PK and safety analyses in the present study should be interpreted in light of several strengths and limitations. Data from the IMbrave150 study are particularly pertinent for the exploration of inter-geographical differences in drug disposition as the predominance of HCC in Asia resulted in IMbrave150 recruiting a higher proportion of 
patients in Asia (close to half of patients on the global trial) than studies in other tumor types. However, one limitation of the study was that the number of patients with severe hepatic impairment per NCI-ODWG criteria was too small for analysis to be conducted in that subgroup. Similarly, no IMbrave150 patients and few GO30140 patients, but no atezolizumab PK-evaluable IMbrave150 patients, were classified as Child-Pugh B7 or ALBI grade 3. Still, we note that a strength of this analysis is the general consistency in results seen across several reported evaluations of liver function, namely, NCIODWG, Child-Pugh, and ALBI. Our findings are important to demonstrate the use of mAbs in a moderate-sized and diverse patient population and provide robust exposure and safety data.

In conclusion, our results demonstrate that neither hepatic impairment as assayed in our study nor enrollment in Asia versus outside Asia had a clinically relevant influence on PK or safety of atezolizumab + bevacizumab. Exposures of each respective agent in the combination therapy were in line with expectations and comparable to previous studies across other indications. The treatment was safe and well-tolerated across evaluated groups, and no dose adjustment is required for differences in mild or moderate hepatic impairment or geographic region. These data extend the primary IMbrave150 results that establish atezolizumab + bevacizumab as a new standard of care for patients with unresectable HCC.

\section{Acknowledgement}

We thank the patients and their families, without whom this study would not have been possible. We acknowledge and thank the investigators and site staff, as well as Dwayne Bracy of Navitas Data Sciences, contracted by Genentech, Inc., for constructing the analysis data set. Medical writing assistance for this manuscript was provided by Ashley J. Pratt, PhD, of Health Interactions and funded by F. Hoffmann-La Roche Ltd.

\section{Statement of Ethics}

Both studies in this analysis were conducted in accordance with the Declaration of Helsinki and the International Conference of Harmonization Good Clinical Practice Guidelines. Protocol approval was obtained from independent review boards (IRBs) or Ethics Committees (ECs) for each participating site. The first IRB approval for IMbrave150 was granted on 19 December 2017 from the City of Hope National Medical Center, Duarte, CA, USA (IRB No. 20172734; Western Institutional Review Board, Inc. Puyallup, WA, USA), and the first IRB approval for GO30140 was granted on 7 March 2016 from the Sarah Cannon Research Institute, Nash-

ville, TN, USA (IRB No. 00008463; IntegReview, Austin, TX, USA). These sites granted approval for each trial in addition to multiple other EC/IRB approvals obtained across all participating sites in 17 and 7 different countries of enrollment for each respective trial. All patients provided written informed consent.

\section{Conflict of Interest Statement}

C.S.S., P.C., D.C., S.V., and B.W. are employees of Genentech, Inc., and Roche stockholders. H.S. and D.X. are employees of F. Hoffmann-La Roche Ltd. and Roche stockholders. R.B. is an employee of Roche France and is a Roche stockholder. D.C. of Combs Consulting Services was contracted by Genentech Inc during the course of the study.

\section{Funding Sources}

This study was sponsored by F. Hoffmann-La Roche Ltd. The sponsor was involved in the design; the collection, analysis, and interpretation of the data; and the writing of the manuscript.

\section{Author Contributions}

C.S.S. wrote the manuscript. All authors assisted with the analysis and provided strategic input. All authors reviewed or contributed to revisions of the manuscript. All authors approved the final manuscript and agreed to be accountable for the work.

\section{Availability of Data and Material}

Qualified researchers may request access to individual patientlevel data through the clinical study data request platform (https:// vivli.org/). Further details on Roche's criteria for eligible studies are available at https://vivli.org/members/ourmembers/. For further details on Roche's Global Policy on the Sharing of Clinical Information and how to request access to related clinical study documents, visit https://www.roche.com/research_and_development/who_we_are_how_we_work/clinical_trials/our_commitment_to_data_sharing.htm.

\section{References}

Liver Cancer 2021;10:485-499

DOI: $10.1159 / 000515817$
1 Keenan BP, Fong L, Kelley RK. Immunotherapy in hepatocellular carcinoma: the complex interface between inflammation, fibrosis, and the immune response. J Immunother Cancer. 2019 Oct 18;7(1):267.

2 Miller KD, Siegel RL, Lin CC, Mariotto AB, Kramer JL, Rowland JH, et al. Cancer treatment and survivorship statistics, 2016. CA Cancer J Clin. 2016 Jul;66(4):271-89.

3 Llovet JM, Zucman-Rossi J, Pikarsky E, Sangro B, Schwartz M, Sherman M, et al. Hepatocellular carcinoma. Nat Rev Dis Primers. 2016 Apr 14;2:16018. 
4 Liu Z, Lin Y, Zhang J, Zhang Y, Li Y, Liu Z, et al. Molecular targeted and immune checkpoint therapy for advanced hepatocellular carcinoma. J Exp Clin Cancer Res. 2019 Nov $4 ; 38(1): 447$.

5 Llovet JM, Ricci S, Mazzaferro V, Hilgard P, Gane E, Blanc JF, et al. Sorafenib in advanced hepatocellular carcinoma. N Engl J Med. 2008 Jul 24;359(4):378-90.

6 Liu Z, Wang Y, Huang Y, Kim BYS, Shan H, $\mathrm{Wu} \mathrm{D}$, et al. Tumor vasculatures: a new target for cancer immunotherapy. Trends Pharmacol Sci. 2019 Sep;40(9):613-23.

7 Hegde PS, Wallin JJ, Mancao C. Predictive markers of anti-VEGF and emerging role of angiogenesis inhibitors as immunotherapeutics. Semin Cancer Biol. 2018 Oct;52(Pt 2): $117-24$.

8 Wallin JJ, Bendell JC, Funke R, Sznol M, Korski $\mathrm{K}$, Jones $\mathrm{S}$, et al. Atezolizumab in combination with bevacizumab enhances antigen-specific T-cell migration in metastatic renal cell carcinoma. Nat Commun. 2016 Aug 30;7: 12624

9 Goel S, Duda DG, Xu L, Munn LL, Boucher Y, Fukumura D, et al. Normalization of the vasculature for treatment of cancer and other diseases. Physiol Rev. 2011 Jul;91(3):1071121.

10 Finn RS, Qin S, Ikeda M, Galle PR, Ducreux M, Kim TY, et al. Atezolizumab plus bevacizumab in unresectable hepatocellular carcinoma. N Engl J Med. 2020 May 14;382(20): 1894-905.

11 Lee MS, Ryoo BY, Hsu CH, Numata K, Stein $\mathrm{S}$, Verret W, et al. Atezolizumab with or without bevacizumab in unresectable hepatocellular carcinoma (GO30140): an open-label, multicentre, phase $1 \mathrm{~b}$ study. Lancet Oncol. 2020 Jun;21(6):808-20.

12 Stroh M, Winter H, Marchand M, Claret L, Eppler S, Ruppel J, et al. Clinical pharmacokinetics and pharmacodynamics of atezolizum$\mathrm{ab}$ in metastatic urothelial carcinoma. Clin Pharmacol Ther. 2017 Aug;102(2):305-12.

13 Morrissey KM, Marchand M, Patel H, Zhang $\mathrm{R}, \mathrm{Wu} \mathrm{B}$, Phyllis Chan $\mathrm{H}$, et al. Alternative dosing regimens for atezolizumab: an example of model-informed drug development in the postmarketing setting. Cancer Chemother Pharmacol. 2019 Dec;84(6):1257-67.

14 Shemesh CS, Chanu P, Jamsen K, Wada R, Rossato G, Donaldson F, et al. Population pharmacokinetics, exposure-safety, and immunogenicity of atezolizumab in pediatric and young adult patients with cancer. J Immunother Cancer. 2019 Nov 21;7(1):314.

15 Shemesh CS, Chan P, Legrand FA, Shames DS, Das Thakur M, Shi J, et al. Pan-cancer population pharmacokinetics and exposuresafety and -efficacy analyses of atezolizumab in patients with high tumor mutational burden. Pharmacol Res Perspect. 2020;8(6) e00685.

16 Lu JF, Bruno R, Eppler S, Novotny W, Lum B, Gaudreault J. Clinical pharmacokinetics of bevacizumab in patients with solid tumors.
Cancer Chemother Pharmacol. 2008 Oct; 62(5):779-86

17 US Food and Drug Administration [Internet]. Guidance for industry. Pharmacokinetics in patients with impaired hepatic function: study design, data analysis, and impact on dosing and labeling [cited 2020 Sep 3]. Available from:https://www.fda.gov/media/71311/ download.

18 Sun Q, Seo S, Zvada S, Liu C, Reynolds K. Does hepatic impairment affect the exposure of monoclonal antibodies? Clin Pharmacol Ther. 2020 May;107(5):1256-62.

19 Kovarik J, Breidenbach T, Gerbeau C, Korn A, Schmidt AG, Nashan B. Disposition and immunodynamics of basiliximab in liver allograft recipients. Clin Pharmacol Ther. 1998 Jul;64(1):66-72.

20 Kovarik JM, Nashan B, Neuhaus P, Clavien PA, Gerbeau C, Hall ML, et al. A population pharmacokinetic screen to identify demographic-clinical covariates of basiliximab in liver transplantation. Clin Pharmacol Ther. 2001 Apr;69(4):201-9.

21 Sheng J, Srivastava S, Sanghavi K, Lu Z, Schmidt BJ, Bello A, et al. Clinical pharmacology considerations for the development of immune checkpoint inhibitors. J Clin Pharmacol. 2017 Oct;57(Suppl 10):S26-42.

22 Forner A, Reig M, Bruix J. Hepatocellular carcinoma. Lancet. 2018 Mar 31;391(10127): 1301-14.

23 Takai T. Roles of Fc receptors in autoimmunity. Nat Rev Immunol. 2002 Aug;2(8):58092.

24 Ishii-Watabe A, Saito Y, Suzuki T, Tada M, Ukaji M, Maekawa K, et al. Genetic polymorphisms of FCGRT encoding FcRn in a Japanese population and their functional analysis. Drug Metab Pharmacokinet. 2010;25(6):57887.

25 Smith KG, Clatworthy MR. Fc $\gamma$ RIIB in autoimmunity and infection: evolutionary and therapeutic implications. Nat Rev Immunol. 2010 May; 10(5):328-43

26 Torkildsen $\varnothing$, Utsi E, Mellgren SI, Harbo HF, Vedeler CA, Myhr KM. Ethnic variation of Fc gamma receptor polymorphism in Sami and Norwegian populations. Immunology. 2005 Jul;115(3):416-21.

27 Matsushima S, Huang Y, Suzuki H, Nishino J, Lloyd P. Ethnic sensitivity assessment - pharmacokinetic comparability between Japanese and non-Japanese healthy subjects on selected mAbs. Expert Opin Drug Metab Toxicol. 2015 Feb;11(2):179-91.

28 Uchida E. [History of the discussion on ethnic difference at ICH]. Yakugaku Zasshi. 2009 Feb;129(2):213-21.

29 Gupta SK. Implications of ICH-E5: assessment of drug's sensitivity to ethnic factors and necessity of a bridging study for global drug development. Perspect Clin Res. 2011 Oct; 2(4):121-3

30 Uyama Y, Shibata T, Nagai N, Hanaoka H, Toyoshima S, Mori K. Successful bridging strategy based on ICH E5 guideline for drugs approved in Japan. Clin Pharmacol Ther. 2005 Aug;78(2):102-13

31 Ling WH, Lee SC. Inter-ethnic differences: how important is it in cancer treatment? Ann Acad Med Singap. 2011 Aug;40(8): 356-61.

32 Zhou H, Tsukamoto Y, Davis HM. Should clinical pharmacokinetic bridging studies between Caucasian and Asian populations be required for approval of monoclonal antibodies? J Clin Pharmacol. 2012 Aug;52(8):12736.

33 Jones PD, Diaz C, Wang D, Gonzalez-Diaz J, Martin P, Kobetz E. The impact of race on survival after hepatocellular carcinoma in a diverse American population. Dig Dis Sci. 2018 Feb;63(2):515-28.

34 Rich NE, Hester C, Odewole M, Murphy CC, Parikh ND, Marrero JA, et al. Racial and ethnic differences in presentation and outcomes of hepatocellular carcinoma. Clin Gastroenterol Hepatol. 2019 Feb;17(3):551-e1.

35 Hoehn RS, Hanseman DJ, Wima K, Ertel AE, Paquette IM, Abbott DE, et al. Does race affect management and survival in hepatocellular carcinoma in the United States? Surgery. 2015 Nov; 158(5):1244-51.

36 Choo SP, Tan WL, Goh BK, Tai WM, Zhu AX. Comparison of hepatocellular carcinoma in Eastern versus Western populations. Cancer. 2016 Nov 15;122(22):3430-46.

37 Younis I. Sensitivity of liver function classification systems for exposure changes. 16th International Workshop on Clinical Pharmacology of HIV and Hepatitis Therapy; 2015 May 26-28; Washington, D.C. [cited 2021 Jan 7]. Available from: http://regist2.virology-education.com/2015/16HIVHEP/03_Younis. pdf.

38 TECENTRIQ. (atezolizumab) [package insert]. South San Francisco, CA: Genentech, Inc; 2020

39 TECENTRIQ. (atezolizumab) [summary of product characteristics]. Welwyn Garden City, UK: Roche Registration Limited; 2020.

40 Nguyen L, Chapel S, Tran BD, Lacy S. Updated population pharmacokinetic model of cabozantinib integrating various cancer types including hepatocellular carcinoma. J Clin Pharmacol. 2019 Nov;59(11):1551-61.

41 Tsoris A, Marlar CA. Use of the Child Pugh score in liver disease. In: Schulz S, Gossman W, Leslie S, Lennox D, Sperduti M, Selvy C, et al., editors. StatPearls [Internet]. Treasure Island, FL: StatPearls Publishing; 2019.

42 Fountzilas C, Stuart S, Hernandez B, BowhayCarnes E, Michalek J, Sarantopoulos J, et al. Risks and benefits of phase I liver dysfunction studies: should patients with severe liver dysfunction be included in these trials? Invest New Drugs. 2017 Jun;35(3):386-91.

43 Johnson PJ, Berhane S, Kagebayashi C, Satomura S, Teng M, Reeves HL, et al. Assessment of liver function in patients with hepatocellular carcinoma: a new evidence-based approach-the ALBI grade. J Clin Oncol. 2015 Feb 20;33(6):550-8. 
44 European Medicines Agency [Internet]. Guideline on the evaluation of the pharmacokinetics of medicinal products in patients with impaired hepatic function [cited 2020 Sep 10]. Available from: https://www.ema.europa.eu/en/documents/scientific-guideline/ guideline-evaluation-pharmacokinetics-medicinal-products-patients-impaired-hepaticfunction_en.pdf.

45 Bergstrand M, Hooker AC, Wallin JE, Karlsson MO. Prediction-corrected visual predictive checks for diagnosing nonlinear mixedeffects models. AAPS J. 2011 Jun;13(2):14351.

46 von Felden J. New systemic agents for hepatocellular carcinoma: an update 2020. Curr Opin Gastroenterol. 2020 May;36(3):177-83.

47 Ryman JT, Meibohm B. Pharmacokinetics of monoclonal antibodies. CPT Pharmacometrics Syst Pharmacol. 2017 Sep;6(9):576-88.

48 Datta-Mannan A, Witcher DR, Tang Y, Watkins J, Wroblewski VJ. Monoclonal antibody clearance. Impact of modulating the interaction of IgG with the neonatal $\mathrm{Fc}$ receptor. J Biol Chem. 2007 Jan 19;282(3):1709-17.

49 Mould DR, Green B. Pharmacokinetics and pharmacodynamics of monoclonal antibodies: concepts and lessons for drug development. BioDrugs. 2010 Feb 1;24(1):23-39.
50 Keizer RJ, Huitema AD, Schellens JH, Beijnen $\mathrm{JH}$. Clinical pharmacokinetics of therapeutic monoclonal antibodies. Clin Pharmacokinet. 2010 Aug;49(8):493-507.

51 Tabrizi MA, Tseng CM, Roskos LK. Elimination mechanisms of therapeutic monoclonal antibodies. Drug Discov Today. 2006 Jan; 11(1-2):81-8.

52 Turner DC, Kondic AG, Anderson KM, Robinson AG, Garon EB, Riess JW, et al. Pembrolizumab exposure-response assessments challenged by association of cancer cachexia and catabolic clearance. Clin Cancer Res. 2018 Dec 1;24(23):5841-9.

53 Han K, Peyret T, Marchand M, Quartino A, Gosselin NH, Girish S, et al. Population pharmacokinetics of bevacizumab in cancer patients with external validation. Cancer Chemother Pharmacol. 2016 Aug;78(2):341-51.

54 Wang E, Song F, Paulus JK, Hackenyos D, Mathew P. Qualitative and quantitative variations in liver function thresholds among clinical trials in cancer: a need for harmonization. Cancer Chemother Pharmacol. 2019 Jul; 84(1):213-6.

55 Elmeliegy M, Yang DZ, Salama E, Parivar K, Wang DD. Discordance between Child-Pugh and National Cancer Institute classifications for hepatic dysfunction: implications on dosing recommendations for oncology compounds. J Clin Pharmacol. 2021 Jul 20;61(1): 105-15.
56 Deng R, Bumbaca D, Pastuskovas CV, Boswell CA, West D, Cowan KJ, et al. Preclinical pharmacokinetics, pharmacodynamics, tissue distribution, and tumor penetration of anti-PD-L1 monoclonal antibody, an immune checkpoint inhibitor. MAbs. 2016;8(3): 593-603.

57 Centanni M, Moes DJAR, Trocóniz IF, Ciccolini J, van Hasselt JGC. Clinical pharmacokinetics and pharmacodynamics of immune checkpoint inhibitors. Clin Pharmacokinet. 2019 Jul;58(7):835-57.

58 Rini BI, Powles T, Atkins MB, Escudier B, McDermott DF, Suarez C, et al. Atezolizumab plus bevacizumab versus sunitinib in patients with previously untreated metastatic renal cell carcinoma (IMmotion151): a multicentre, open-label, phase 3, randomised controlled trial. Lancet. 2019 Jun 15;393(10189): 2404-15.

59 Socinski MA, Jotte RM, Cappuzzo F, Orlandi F, Stroyakovskiy D, Nogami N, et al. Atezolizumab for first-line treatment of metastatic nonsquamous NSCLC. N Engl J Med. 2018 Jun 14;378(24):2288-301.

60 Zhou L, Higashimori M, Shen K, Zhang Z, Sheng J, Xu H, et al. Oncology therapy drugs in China, Japan, and the United States: pharmacokinetic characteristics, dose regimens, and development strategies. Clin Pharmacol Ther. 2019 Jun;105(6):1303-20. 